\title{
Influence of Dietary Sesamin, a Bioactive Compound on Fatty Acids and Expression of Some Lipid Regulating Genes in Baltic Atlantic Salmon (Salmo salar L.) Juveniles
}

\author{
S. TRATTNER ${ }^{1,3}$, B. RUYTER ${ }^{2}$, T. K. ØSTBYE ${ }^{2}$, A. KAMAL-ELDIN ${ }^{1}$, A. MOAZZAMI ${ }^{1}$, \\ J. PAN ${ }^{1}$, T. GJØEN ${ }^{4}$, E. BRÄNNÄS ${ }^{3}$, V. ZLABEK ${ }^{1,5}$, J. PICKOVA ${ }^{1,5}$ \\ ${ }^{1}$ Department of Food Science, SLU, Uppsala, Sweden, ${ }^{2}$ Nofima AS, Ås, Norway, ${ }^{3}$ Department of \\ Wildlife, Fish, and Environmental Studies, SLU, Umeå, Sweden, ${ }^{4}$ Department of Pharmaceutical \\ Bioscience, School of Pharmacology, University of Oslo, Oslo, Norway, ${ }^{5}$ Research Institute of Fish \\ Culture and Hydrobiology, University of South Bohemia in Ceske Budejovice, Vodnany, Czech \\ Republic
}

Received August 20, 2010

Accepted September 21, 2010

On-line October 15, 2010

\section{Summary}

The effects of inclusion of sesamin / episesamin in Baltic Atlantic salmon (Salmo salar L.) diets based on vegetable oils were studied. The study was designed as a dose response study with two control diets, one diet based on fish oil (FO) and one diet based on a mixture of linseed and sunflower oil (6:4 by vol.) (MO). As experimental diets three different levels of inclusion of sesamin / episesamin (hereafter named sesamin) to the MO based diet and one diet based on sesame oil and linseed oil (SesO) (1:1 by vol.) were used. The dietary oils were mirrored in the fatty acid profile of the white muscle. Sesamin significantly decreased the levels of $18: 3 n-3$ in the white muscle phospholipid $(\mathrm{PL})$ fraction of all groups fed sesamin, no significant differences were found in the triacylglycerol fraction (TAG). Slightly increased levels of docosahexaenoic acid (22:6n-3, DHA) in PL and TAG were found in some of the sesamin fed groups. Sesamin significantly affected the expression of peroxisome proliferatoractivated receptor $a$, scavenger receptor type $B$ and hormone sensitive lipase, in agreement with previous studies on rainbow trout (Oncorhynchus mykiss) and Atlantic salmon (Salmo salar L.) hepatocytes published by our group. No significant effects on toxicological response measured as ethoxyresorufin O-deethylase activity was found. The total cytochrome P450 enzymes were significantly higher in MO 0.29 and SesO group. The amount of $\mathrm{a}$ - and $\mathrm{y}$-tocopherols in liver and the amount of $\mathrm{y}$-tocopherol in white muscle were significantly lower in fish fed the FO diet compared to the MO diet, but no difference after inclusion of sesamin was found in this study. Increased inclusion of sesamin increased the levels of sesamin and episesamin in the liver, but did not affect the amounts in white muscle.

\section{Key words}

Sesamin - Episesamin - Tocopherols - Cytochrome P450 • Peroxisome proliferator-activated receptor - Hormone sensitive lipase • Scavenger receptor type $B$

\section{Corresponding author}

Sofia Trattner, Department of Food Science, SLU, Uppsala, Sweden. Fax: 46-18-672995. E-mail: Sofia.Trattner@Imv.slu.se

\section{Introduction}

Sesamin, a minor component of sesame oil (Moazzami and Kamal-Eldin 2006), is a potent lipid modulator in mammals. It has been shown to affect enzymatic activity and expression of genes involved in lipid metabolism e.g. acyl-CoA oxidase (ACO) and carnitine palmitoyltransferase (CPT) (Kushiro et al. 2002, Jeng and Hou 2005, Kiso et al. 2005). In the fungus Mortierella alpina and in primary rat hepatocytes, sesamin was shown to reduce $\Delta-5$ desaturation index and enzymatic activity of $\Delta-5$ desaturase (Shimizu et al. 1991). To our knowledge, only two studies from our group have investigated the effects of dietary sesamin in 
fish. In these studies, it was shown that sesamin increased docosahexaenoic acid (22:6n-3, DHA) in rainbow trout (Oncorhynchus mykiss) white muscle phospholipid (PL) and triacylglycerol (TAG) fraction and decreased the expression of peroxisome proliferator-activated receptor $\alpha(\operatorname{PPAR} \alpha)$ in liver (Trattner et al. 2008a). In Atlantic salmon (Salmo salar L.) hepatocytes, it was shown that sesamin increased elongation and desaturation of radiolabelled 18:3n-3 towards DHA. It increased the levels of $\beta$-oxidation products and the relative expression of cluster of differentiation 36 (CD36), scavenger receptor (SRB) type $\mathrm{B}, \mathrm{PPAR} \alpha$ and $\gamma$ (Trattner et al. 2008b). The metabolic effects of sesamin have been suggested to be caused through the activation of PPARs and sterol regulatory element binding protein-1 (SREBP1) (Ashakumary et al. 1999, Ide et al. 2004). Furthermore, sesamin has been reported to inhibit cholesterol absorption and synthesis, and tocopherol hydroxylation and clearance in rats and humans (Jeng and Hou 2005). Other compounds known to modulate lipids are 3-thia fatty acids, conjugated linoleic acid and Lipoic acid (Berge et al. 2001, Huong and Ide 2008, Kennedy et al. 2009).

The methylenedioxyphenyl group of sesamin is known to affect cytochrome P450-dependent drug oxidation (Murray 2000). Cytochrome P450 (CYP) enzymes are known to play a central role in the oxidative metabolism and biotransformation of a wide range of endogenous and exogenous compounds (Nelson et al. 1996). Among the numerous CYP families identified, primarily CYP 1-3 are involved in biotransformation of xenobiotics. The CYP1A subfamily is reported to be expressed in the liver of both mammals and fish (Murray 2000, Jönsson et al. 2006). Due to the role of CYP1A isoenzymes in the metabolism and bioactivation of foreign compounds, alteration of the expression of hepatic CYP1A may affect the potential risk of xenobiotics (Williams et al. 1998). CYP1A is readily inducible by aryl hydrocarbon (Ah) receptor agonist, thus the activity of CYP1A, measured as ethoxyresorufin Odeethylase (EROD) activity, is used as a biomarker for exposure to xenobiotic compounds in fish (Havelkova et al. 2007).

Traditionally, carnivorous farmed fish has been fed diets based on fish ingredients. At present there is an overuse of marine raw materials for aquaculture feed production and at the same time aquaculture is the fastest growing food production industry (Tacon 2005, FAO 2007). Therefore, alternative fish feed ingredients are being investigated. Vegetable oil is used as a replacement of fish oil (up to $50 \%$ ) without affecting growth and production yield (Torstensen et al. 2005). One well known drawback of replacement with vegetable oils in fish feed are the decreased amounts of n-3 highly unsaturated fatty acids (HUFA) in fish tissues (Torstensen et al. 2005, Pettersson et al. 2009). The n-3 HUFA are known to have positive health effects in man. In terms of human health, it is important to preserve the beneficial fatty acid (FA) composition of fish (Mozaffarian and Rimm 2006). It is necessary to find alternatives to fish oil use, without decreasing the content of n-3 HUFA in fish.

To achieve more n-3 HUFA in fish fed vegetable oils, bioactive compounds can be added in the fish diet. It is interesting to study the effects on sesamin in common aquaculture species, on the nutritional quality of muscle as human food in terms of FA, tocopherols and sesamin content. Furthermore, it is important to investigate how sesamin is metabolized in the fish and how it affects fish welfare. To investigate the effects of sesamin in wild strain Baltic Atlantic salmon (Salmo salar L.) juveniles, a dose response study was designed and the FA composition, the relative expression of nine lipid related genes, the content of tocopherols, sesamin and episesamin, EROD activity and the total level of CYP were analyzed.

\section{Material and Methods}

\section{Chemicals and reagents}

Sesamin/episesamin mixture $(1: 1, \mathrm{w} / \mathrm{w})$ was a kind gift from Takemoto Oil and Fat Co., Ltd. (Gamagori Aichi, Japan). Fatty acid peaks were identified by comparison with the standard mixture GLC-68 A (Nucheck Prep, Inc, Elysian, Minnesota, USA). Tocopherol standards were purchased as an isomer kit (article number 15496) from Merck (Darmstadt, Germany). All solvents and other chemicals for FA, tocopherols and sesamin analysis were also purchased from Merck and were used without further purification.

\section{Animals and diets}

Baltic Atlantic salmon was fed six different diets, fifteen fish per group. Prior the experiment all fish were fed the same commercial diet. Four groups were fed experimental diets based of vegetable oils and sesamin/episesamin mixture $(1: 1, \mathrm{w} / \mathrm{w})$ (hereafter named sesamin), while one group was fed a diet based on fish 
ingredients, and one group was fed a diet based on vegetable oil. The diets were prepared according to the method of Sanchez-Vazquez (1999), the ingredients are shown in Table 1. The diets differed in oil composition and the content of sesamin as follow; 1) mixed linseed : sunflower oil, 6:4 by vol. (MO), 2) fish oil (FO), 3) sesame oil : linseed oil, 1:1 by vol. ( $\mathrm{SesO}$ ), 4) MO +

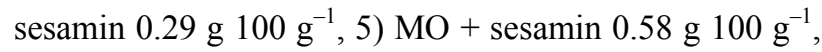

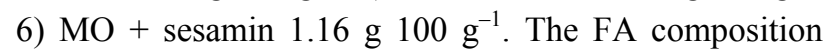
and the tocopherol content of the diets are shown in Table 2. The fish were tagged individually and the individual weight increase was calculated as daily growth gain. Fishes were kept at a water temperature of $10{ }^{\circ} \mathrm{C}$ and were fed ad libitum for 77 days. Before the experiment started, the fish were tagged with a PIT-tag (Passive Integrated Transponder) by injecting the tag into the posterior part of the abdomen. Before handling, all fish were anaesthetized (ethyleneglycol monophenyl ether $5 \mathrm{ml} \mathrm{l}^{-1}$ ). The daily growth rate (DGC) were calculated as: $\mathrm{DGC}=100 \times\left(\mathrm{W}_{2}^{1 / 3}-\mathrm{W}_{1}{ }^{1 / 3}\right) \mathrm{D}^{-1}$ with $\mathrm{W}_{2}$ being final weight, $\mathrm{W}_{1}$ the starting weight and $D$ the number of days (Table 3.).

Table 1. Basic feed ingredients in the experimental diets.

\begin{tabular}{lc}
\hline Ingredient & $\mathbf{g ~} \mathbf{1 0 0} \mathbf{~ g}^{-\mathbf{1}}$ \\
\hline Casein & 17.7 \\
Gelatin & 3.0 \\
Fish meal & 20.7 \\
Dextrin & 9.3 \\
Oil ${ }^{*}$ & 27.0 \\
Vitamins + minerals & 0.3 \\
Ca $_{3} \mathrm{PO}_{4}$ & 3.8 \\
Cellulose $_{\text {Na alginate }}$ & 14.3 \\
\hline
\end{tabular}

* The oil used was a mixture of linseed and sunflower oil $(6: 4)$ in the MO diets, in the SesO diet sesame oil : linseed oil (1:1) was used and in the FO diet fish oil was used. Sesamin / episesamin

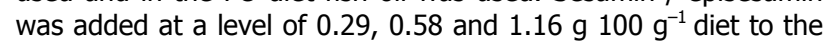
MO 0.29, MO 0.58 and MO 1.16 diet.

At sacrifice, the muscle was divided in red and white. The muscles, intestine and liver were frozen at $-80{ }^{\circ} \mathrm{C}$ until analyzed. From each group six individuals were used for fatty acid, tocopherols, EROD and CYP analysis and another six individuals were used for gene expression analysis.

\section{Lipid analysis}

White muscle $(2 \mathrm{~g})$ and diets $(1 \mathrm{~g})$ were extracted following the method of (Hara and Radin 1978). The lipid content was measured gravimetrically. Total lipids of tissues were separated into PL and TAG according to Pickova et al. (1997). Total lipids in the diets, and the PL and TAG lipid fractions of tissues were methylated following the procedure of (Appelqvist 1968) and the FA were analysed by gas chromatograph CP3800 (Varian AB, Stockholm, Sweden) equipped with flame ionisation detector (FID) and split injector and fitted with a fused silica capillary column BPX 70 (SGE, Austin, Tex.), length $50 \mathrm{~m}$, id. $0.22 \mathrm{~mm}, 0.25 \mu \mathrm{m}$ film thickness. The column temperature was programmed to start at $158^{\circ} \mathrm{C}$ hold 5 min and then increase $2{ }^{\circ} \mathrm{C} / \mathrm{min}$ from $158^{\circ} \mathrm{C}$ to $220^{\circ} \mathrm{C}$ and remain at $220^{\circ} \mathrm{C}$ for $8 \mathrm{~min}$. The carrier gas was helium $(0.8 \mathrm{ml} /$ min) and make up gas was nitrogen. The injector and detector temperatures were $230{ }^{\circ} \mathrm{C}$ and $250{ }^{\circ} \mathrm{C}$, respectively. FA were identified by comparison with the standard FA mixture GLC-68. Peak areas were integrated using Varian Star chromatography workstation software version 5.5.

\section{Tocopherols, sesamin and episesamin analyses}

For the analysis of tocopherols in the diets, and the tocopherols, sesamin and episesamin in the tissues, the lipid extracts were dissolved in hexane and analysed with high performance liquid chromatography (HPLC). The mobile phase used was hexane/1,4-dioxane (94:4, vol/vol). The HPLC system was equipped with a Bischoff HPLC pump (Bischoff Analysentechnik und geräte $\mathrm{GmbH}$, Leonberg, Germany) and Agilent 1100 series fluorescence detector (Agilent Technologies, Waldbronn, Germany). The HPLC column was Alltech SI $5 \mathrm{U}$ silica column $(4.6 \times 250 \mathrm{~mm}$; Alltech Associates Inc., Deerfield, IL). The fluorescence detector was operated at an excitation wavelength of $296 \mathrm{~nm}$ and an emission wavelength of $324 \mathrm{~nm}$ as described by Moazzami and Kamal-Eldin (2006). Identification and quantification was achieved by comparison to external standards.

\section{RNA analysis}

Total RNA was purified from livers, muscle and intestine from each group $(n=6)$ and analyzed in duplicate, using Trizol ${ }^{\circledR}$ (Invitrogen), followed by DNase treatment (TURBO DNA-free, Ambion). All protocols were according to the manufacturer's instructions. RNA 
Table 2. Fatty acid composition of the diets, ( $\%$ of total FA), and tocopherols $(\mu \mathrm{g} / \mathrm{g}$ lipid), duplicate analyses.

\begin{tabular}{|c|c|c|c|c|c|c|}
\hline & MO & FO & SesO & MO 0.29 & MO 0.58 & MO 1.16 \\
\hline $14: 0$ & 0.22 & 7.4 & 0.18 & 0.20 & 0.19 & 0.19 \\
\hline $16: 0$ & 6.47 & 17.2 & 8.13 & 6.30 & 6.17 & 6.16 \\
\hline $16: 1$ & 0.18 & 6.88 & 0.20 & 0.18 & 0.17 & 0.17 \\
\hline $18: 0$ & 3.46 & 2.28 & 4.87 & 3.49 & 3.49 & 3.50 \\
\hline $18: \ln -9$ & 21.3 & 11.8 & 28.0 & 21.3 & 21.3 & 21.2 \\
\hline $18: 1 n-7$ & 0.65 & 2.22 & 0.73 & 0.65 & 0.64 & 0.65 \\
\hline $18: 2 n-6$ & 33.7 & 1.66 & 29.7 & 33.7 & 33.7 & 33.6 \\
\hline $18: 3 n-3$ & 30.0 & 1.31 & 24.8 & 29.9 & 29.9 & 29.9 \\
\hline $20: 1$ & 0.22 & 6.00 & 0.23 & 0.23 & 0.22 & 0.23 \\
\hline $22: 1$ & 0.05 & 9.55 & 0.11 & 0.11 & 0.05 & 0.00 \\
\hline $20: 5 n-3$ & 0.16 & 8.38 & 0.16 & 0.15 & 0.15 & 0.17 \\
\hline $22: 5 n-3$ & 0.00 & 0.78 & n.d & n.d & n.d & n.d \\
\hline $22: 6 n-3$ & 0.32 & 9.79 & 0.34 & 0.34 & 0.31 & 0.36 \\
\hline$S A F A$ & 10.5 & 27.2 & 13.7 & 10.6 & 10.4 & 10.3 \\
\hline$M U F A$ & 22.5 & 37.4 & 29.2 & 22.6 & 22.6 & 22.5 \\
\hline$P U F A$ & 64.3 & 22.8 & 55.1 & 64.1 & 64.1 & 64.2 \\
\hline$n-3$ & 30.5 & 20.4 & 25.3 & 30.4 & 30.4 & 30.5 \\
\hline$n-6$ & 33.9 & 2.40 & 29.8 & 33.7 & 33.7 & 33.8 \\
\hline$n-3 / n-6$ & 0.90 & 8.49 & 0.85 & 0.90 & 0.90 & 0.90 \\
\hline$\alpha$-tocopherol & 1900 & 600 & 1000 & 2200 & 2500 & 2100 \\
\hline$\gamma$-tocopherol & 2300 & n.d & 4000 & 2300 & 2600 & 2500 \\
\hline
\end{tabular}

SAFA $=$ saturated fatty acids $(20: 0,20: 2,22: 0,24: 0)$, MUFA = monounsaturated fatty acids $(14: 1,18: 1$ n-5, 24:1), PUFA = polyunsaturated fatty acids. $\mathrm{MO}=$ mixed oil, $\mathrm{FO}=$ fish oil, $\mathrm{SesO}=$ sesame oil : linseed oil, $\mathrm{MO} 0.29=$ mixed oil with sesamin addition

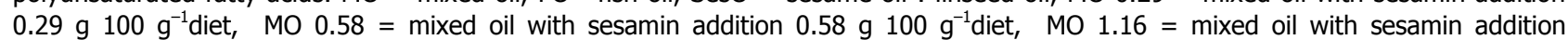
$1.16 \mathrm{~g} 100 \mathrm{~g}^{-1}$ diet.

Table 3. Daily growth coefficient $(n=6)$ and range between the smallest and largest value during 77 days feeding period.

\begin{tabular}{lcccccc}
\hline & FO & SesO & MO & MO 0.29 & MO 0.58 & MO 1.16 \\
\hline $\begin{array}{l}\text { Average } \pm \text { StDev } \\
\text { Range }\end{array}$ & $0.9 \pm 0.14$ & $0.9 \pm 0.28$ & $0.8 \pm 0.20$ & $0.7 \pm 0.28$ & $0.7 \pm 0.14$ & $0.7 \pm 0.10$ \\
$0.66-1.03$ & $0.54-1.20$ & $0.53-1.11$ & $0.29-1.16$ & $0.50-0.91$ & $0.55-0.84$ \\
\hline
\end{tabular}

Abbreviations see Table 2.

quality and quantity were determined spectrophotometrically $\left(\mathrm{A}_{260 / 280}\right)$ using NanoDrop® (ND-1000 Spectrophotometer, NanoDrop Technologies, Wilmington, Delaware, USA). Samples were stored in RNase-free water at $-80^{\circ} \mathrm{C}$.

The cDNA was synthesized from $2.4 \mu \mathrm{g}$ RNA, a modified protocol from the Taq Man Reverse Transcription Reagents kit (Applied Biosystems). The Oligo $\mathrm{d}(\mathrm{T})_{16}$ primers were used. The reaction was performed by incubating the samples at $25^{\circ} \mathrm{C}$ for $10 \mathrm{~min}$, $48^{\circ} \mathrm{C}$ for $6 \mathrm{~min}, 95^{\circ} \mathrm{C}$ for $50 \mathrm{~min}$ and was terminated by reducing the temperature to $10^{\circ} \mathrm{C}$. Primers for Real-Time PCR analysis (Table 4) were designed using the Primer Express ${ }^{\circledR}$ software based on available salmon sequences in the GenBank ${ }^{\circledR}$ and purchased from Invitrogen (CA, USA). Real-Time PCR was performed in a Prism ${ }^{\circledR} 7000$ system by using gene-specific primers. A 2 x SYBR $\mathbb{R}$ Green PCR Mastermix (ABI) was used in the PCR reaction mix of $25 \mu \mathrm{l}$ with $1 \mu \mathrm{l}$ primers (final concentration of $0.5 \mu \mathrm{M}$ ), and $5 \mu \mathrm{l}$ cDNA. All samples 
Table 4. Sequences of primers used for real time PCR analysis.

\begin{tabular}{lllc}
\hline Primer & Forward primer (5'-3') & Reverse primer (5'-3') & Efficiency \\
\hline$R P L 2$ & TAACGCCTGCCTCTTCACGTTGA & ATGAGGGACCTTGTAGCCAGCAA & 1.95 \\
$E F 1 A$ & CACCACCGGCCATCTGATCTACAA & TCAGCAGCCTCCTTCTCGAACTTC & 1.97 \\
$P P A R \alpha$ & CGTTGAATTTCATGGCGAACT & TCCTGGTGGCCTACGGATC & 1.90 \\
$P P A R \beta$ & CCAGCAACCCGTCCTTGTT & GAGACGGTCAGGGAGCTCAC & 2.04 \\
$P P A R \gamma$ & CATTGTCAGCCTGTCCAGAC & ATGTGACATTCCCACAAGCA & 1.95 \\
$S R B-I$ & AACTCAGTGAAGAGGCCAAACTTG & TGCGGCGGTGATGATG & 1.79 \\
$C D 36$ & GGATGAACTCCCTGCATGTGA & TGAGGCCAAAGTACTCGTCGA & 1.76 \\
$H S L 3$ & AACGTAGATCAGCCAGTCACCC & ACGTTAGCCGCTTCCCTAGTCT & 1.88 \\
$\Delta 5$ & GAGAGCTGGCACCGACAGAG & GAGCTGCATTTTTCCATGG & 1.77 \\
$\Delta 6$ & AGAGCGTAGCTGACACAGCG & TCCTCGGTTCTCTCTGCTCC & 1.90 \\
\hline
\end{tabular}

RPL2 = RNA polymerase II polypeptide, EF1A = Elongation factor 1a, PPAR = peroxisome proliferator-activated receptor, SRB-I = scavenger receptor type $B, C D 36=$ cluster of differentiation $36, \mathrm{HSL3}=$ hormone sensitive lipase, $\Delta 5=\Delta 5$ desaturase, $\Delta 6=\Delta 6$ desaturase.

were analyzed in duplicate with a non-template control on each plate. The reference genes used were elongation factor $1 \alpha$ (EF1A) and RNA polymerase II polypeptide (RPL II). The reaction was performed by incubating the samples at $50{ }^{\circ} \mathrm{C}$ for $2 \mathrm{~min}, 95^{\circ} \mathrm{C} 10 \mathrm{~min}$ and 50 cycles of $95{ }^{\circ} \mathrm{C}$ for $10 \mathrm{~s}$ and $60{ }^{\circ} \mathrm{C}$ for $15 \mathrm{~s}$. Standard curves were made for each primer pair and efficiencies (E) were calculated $\mathrm{E}=10^{(-1 / \text { slope })}$

\section{Total content of CYP and EROD activity in liver}

For the analysis of total CYP content and EROD activity, six liver samples from each group were analysed. The liver tissue was homogenized in ice-cold homogenization buffer $(0.25 \mathrm{M}$ sucrose and $0.1 \mathrm{mM}$ EDTA in $0.01 \mathrm{M}$ TRIS buffer, $\mathrm{pH}$ 7.4) using a PotterElvehjem homogeniser. The homogenate was centrifuged for $15 \mathrm{~min}$ at $10,000 \times \mathrm{g}\left(4^{\circ} \mathrm{C}\right)$ and the resulting supernatant was spun down for $1 \mathrm{~h}$ at $105,000 \times g\left(4^{\circ} \mathrm{C}\right)$. The microsomal pellets were resuspended in the homogenization buffer and stored at $-80{ }^{\circ} \mathrm{C}$ until used. The total CYP content was determined spectrophotometrically by the Co- and dithionite difference method (Shimanzu UV-1601PC, Columbia, USA) according to (Omura and Sato 1964).

Hepatic EROD activity was determined according to a modified method (Jönsson et al. 2006). Standard solutions of resorufin $(0-50 \mu \mathrm{M})$ and protein

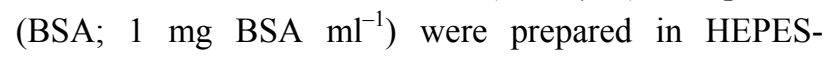
Cortland buffer $\mathrm{pH} 8$. The $\mathrm{HC}$ buffer was prepared by dissolving $0.38 \mathrm{~g} \mathrm{KCl}, 7.74 \mathrm{~g} \mathrm{NaCl}, 0.23 \mathrm{~g} \mathrm{MgSO}_{4} \cdot 7 \mathrm{H}_{2} \mathrm{O}$, $0.23 \mathrm{~g} \mathrm{CaCl}_{2} \cdot 2 \mathrm{H}_{2} \mathrm{O}, 0.41 \mathrm{~g} \quad \mathrm{NaH}_{2} \mathrm{PO}_{4} \cdot \mathrm{H}_{2} \mathrm{O}, 1.43 \mathrm{~g}$
HEPES, and $1 \mathrm{~g}$ glucose in 11 of distilled water. Microsome suspensions were further diluted in the same buffer (1:5 and 1:10). Aliquots of the microsome suspensions $(50 \mu 1)$ and of the standard solutions $(40 \mu 1$ of resorufin and $10 \mu 1$ of BSA) were added in duplicate wells in 96-well plate. A $160 \quad \mu 1$ aliquot of 7-ethoxyresorufin $(12.7 \mu \mathrm{M})$ and NADPH $(2.1 \mathrm{mM})$ in $\mathrm{HC}$ buffer was rapidly added to all wells. The plate was then immediately placed in a microplate reader (Wallac 1420 VICTOR $^{2}$, Turku, Finland) and the resorufin fluorescence was monitored for $10 \mathrm{~min}$ by repeated measurements at $544 \mathrm{~nm}(\mathrm{ex})$ and $590 \mathrm{~nm}(\mathrm{em})$. EROD activity was calculated and expressed as pmol of resorufin formed per mg protein and minute. The protein contents of the microsomes were assayed by the (Smith et al. 1985), adapted for microplate readers.

Data analysis

Fatty acids, EROD, CYP, tocopherols, sesamin and episesamin data are presented as mean values \pm standard deviation. The General Linear Model (GLM) of SAS (SAS Institute Inc., Cary, N.C., USA, version 8.2) was used to compare the physiological responses of the different diets. The model included the fixed effect of treatment and random effect of individual. Relative expression of the different genes, in relation to housekeeping genes were determined by using the Relative Expression Software Tool (REST-384C-version 1) for group wise comparison and statistical analysis of relative expression results in real-time PCR (Pfaffl et al. 2002). 


\section{Results}

\section{Survival}

The total mortality during the study was 5 fish, of which three belonged to the FO-group, one to the MO group and one to the MO 0.28 group.

There was no difference in starting weight, final weight or daily growth coefficient (DGC) between the MO group and the groups with added sesamin. For the FO group both start and end weight were lower (6.9 and 13.8 , respectively) than in the other groups (range 8.7$20.7 \mathrm{~g}$ ). The DGR is shown for the six fish from each group which were analysed for fatty acid composition. The variation between individuals is large in all groups as seen from the data in Table 3 .

Fatty acid composition and lipid content

The groups did not differ in lipid content. The
FA composition of white muscle clearly reflected the FA profile of the oils used in the diets (Table 5). The FO group was characterized by high proportions of EPA and DHA in PL and TAG. The vegetable oil groups had higher proportions of 18:3n-3 and lower proportions of n-3HUFA. The response to the change in dietary FA composition was faster in TAG than PL. The levels of 18:3n-3 were significantly lower in PL of all three groups fed sesamin compared to the MO group without sesamin $(P<0.05)$. The level of docosapentaenoic acid (DPA, 22:5n-3) and DHA in PL of the MO groups with added sesamin were higher than in the MO group, however the difference was only significant for DPA in the MO 0.58 group $(P<0.05)$. In the PL of MO 0.58 group DHA increased to $36.6(P=0.16)$. Similar to the PL fraction 18:3n-3 decreased and DPA and DHA increased in the TAG of MO 0.58 and MO 1.16 group (non significant changes).

Table 5. Fatty acid composition of the white muscle PL and TAG fraction, duplicate analyses, $n=6$ ( $\%$ of total FA).

\begin{tabular}{|c|c|c|c|c|c|c|}
\hline & FO & SesO & MO & М 0.29 & M 0.58 & MO 1.16 \\
\hline Lipid (\%) & $2.00 \pm 0.43$ & $2.06 \pm 0.54$ & $1.92 \pm 0.55$ & $1.74 \pm 0.33$ & $1.82 \pm 0.51$ & $1.92 \pm 0.31$ \\
\hline \multicolumn{7}{|l|}{ Phospholipids } \\
\hline $14: 0^{1}$ & $1.52 \pm 0.26^{\mathrm{Z}}$ & $0.41 \pm 0.09^{\mathrm{y}}$ & $0.52 \pm 0.19^{z}$ & $0.40 \pm 0.03^{z}$ & $0.41 \pm 0.09^{\mathrm{z}}$ & $0.43 \pm 0.03^{\mathrm{z}}$ \\
\hline 16:0 & $17.6 \pm 5.28^{z}$ & $16.1 \pm 1.46^{\mathrm{zy}}$ & $16.9 \pm 1.19^{\mathrm{zy}}$ & $14.5 \pm 1.19^{y}$ & $15.5 \pm 1.33^{\mathrm{zy}}$ & $15.8 \pm 1.15^{\mathrm{zy}}$ \\
\hline $16: 1$ & $1.75 \pm 0.21$ & $0.34 \pm 0.03$ & $0.45 \pm 0.21$ & $0.36 \pm 0.02$ & $0.37 \pm 0.08$ & $0.40 \pm 0.04$ \\
\hline 18:0 & $4.05 \pm 0.21^{\mathrm{z}}$ & $5.85 \pm 0.47^{y}$ & $5.59 \pm 0.31^{\mathrm{y}}$ & $5.69 \pm 0.28^{y}$ & $5.76 \pm 0.40^{\mathrm{y}}$ & $5.88 \pm 0.26^{\mathrm{y}}$ \\
\hline $18: 1 n-9$ & $7.05 \pm 1.21^{z}$ & $9.70 \pm 2.79^{y}$ & $7.68 \pm 1.21^{z}$ & $7.29 \pm 0.82^{z}$ & $7.40 \pm 1.07^{\mathrm{z}}$ & $6.98 \pm 0.76^{z}$ \\
\hline $18: 1 n-7$ & $2.40 \pm 0.14^{\mathrm{z}}$ & $1.20 \pm 0.18^{\mathrm{y}}$ & $1.28 \pm 0.11^{\mathrm{y}}$ & $1.21 \pm 0.04^{\mathrm{y}}$ & $1.33 \pm 0.06^{\mathrm{y}}$ & $1.30 \pm 0.11^{\mathrm{y}}$ \\
\hline $18: 1 n-5$ & $0.52 \pm 0.08$ & $0.53 \pm 0.18$ & $0.44 \pm 0.04$ & $0.56 \pm 0.14$ & $0.43 \pm 0.02$ & $0.50 \pm 0.12$ \\
\hline $18: 2 n-6$ & $1.25 \pm 0.24^{\mathrm{z}}$ & $8.53 \pm 0.99^{y}$ & $8.46 \pm 0.49^{y}$ & $8.59 \pm 0.87^{y}$ & $8.05 \pm 0.31^{\mathrm{y}}$ & $8.37 \pm 0.65^{\mathrm{y}}$ \\
\hline $18: 3 n-3$ & $0.68 \pm 0.12^{z}$ & $5.55 \pm 0.50^{\mathrm{x}}$ & $6.45 \pm 0.53^{y}$ & $5.81 \pm 0.28^{\mathrm{x}}$ & $5.77 \pm 0.22^{x}$ & $5.97 \pm 0.32^{\mathrm{x}}$ \\
\hline $20: 0$ & $0.11 \pm 0.12$ & $0.12 \pm 0.03$ & $0.07 \pm 0.04$ & $0.09 \pm 0.01$ & $0.08 \pm 0.04$ & $0.07 \pm 0.02$ \\
\hline $18: 4 n-3$ & $0.66 \pm 0.27^{z}$ & $2.24 \pm 0.46^{\mathrm{y}}$ & $2.16 \pm 0.34^{\mathrm{y}}$ & $2.05 \pm 0.43^{\mathrm{y}}$ & $1.87 \pm 0.21^{\mathrm{y}}$ & $2.07 \pm 0.09^{y}$ \\
\hline $20: 1$ & $0.53 \pm 0.45^{z}$ & $0.26 \pm 0.13^{\mathrm{y}}$ & $0.25 \pm 0.20^{\mathrm{y}}$ & $0.20 \pm 0.09^{\mathrm{y}}$ & $0.21 \pm 0.11^{\mathrm{y}}$ & $0.18 \pm 0.03^{\mathrm{y}}$ \\
\hline $20: 2 n-6$ & $0.17 \pm 0.03^{z}$ & $0.43 \pm 0.04^{\mathrm{y}}$ & $0.36 \pm 0.03^{\mathrm{y}}$ & $0.44 \pm 0.06^{\mathrm{y}}$ & $0.43 \pm 0.04^{\mathrm{y}}$ & $0.40 \pm 0.07^{\mathrm{y}}$ \\
\hline $20: 3 n-6$ & $0.07 \pm 0.03^{z}$ & $0.89 \pm 0.10^{\mathrm{y}}$ & $0.67 \pm 0.08^{\mathrm{x}}$ & $0.87 \pm 0.04^{\mathrm{yu}}$ & $0.77 \pm 0.06^{\mathrm{v}}$ & $0.79 \pm 0.09^{\mathrm{vu}}$ \\
\hline $20: 4 n-6$ & $1.51 \pm 0.21^{\mathrm{z}}$ & $1.47 \pm 0.14^{\mathrm{z}}$ & $1.31 \pm 0.19^{\mathrm{y}}$ & $1.51 \pm 0.11^{\mathrm{z}}$ & $1.44 \pm 0.10^{z}$ & $1.50 \pm 0.08^{\mathrm{z}}$ \\
\hline $20: 3 n-3$ & $0.09 \pm 0.02^{\mathrm{z}}$ & $0.38 \pm 0.06^{\mathrm{yx}}$ & $0.35 \pm 0.03^{y}$ & $0.43 \pm 0.07^{\mathrm{x}}$ & $0.42 \pm 0.07^{\mathrm{yx}}$ & $0.40 \pm 0.08^{\mathrm{yx}}$ \\
\hline $22: 1$ & $1.02 \pm 0.12$ & $1.30 \pm 0.05$ & $1.21 \pm 0.15$ & $1.36 \pm 0.13$ & $1.28 \pm 0.12$ & $1.28 \pm 0.11$ \\
\hline $20: 5 n-3$ & $8.99 \pm 0.84^{z}$ & $5.87 \pm 0.46^{\mathrm{y}}$ & $6.38 \pm 0.67^{y}$ & $6.34 \pm 0.48^{\mathrm{y}}$ & $6.14 \pm 0.26^{\mathrm{y}}$ & $6.39 \pm 0.23^{y}$ \\
\hline $24: 1$ & $0.16 \pm 0.05$ & $0.11 \pm 0.05$ & $0.12 \pm 0.08$ & $0.08 \pm 0.02$ & $0.13 \pm 0.05$ & $0.07 \pm 0.04$ \\
\hline $22: 5 n-3$ & $1.80 \pm 0.31^{\mathrm{zx}}$ & $1.30 \pm 0.21^{\mathrm{yx}}$ & $1.26 \pm 0.10^{\mathrm{y}}$ & $1.39 \pm 0.11^{\mathrm{yx}}$ & $1.48 \pm 0.10^{\mathrm{x}}$ & $1.38 \pm 0.16^{\mathrm{yx}}$ \\
\hline $22: 6 n-3$ & $40.7 \pm 2.55^{z}$ & $33.2 \pm 4.71^{\mathrm{y}}$ & $34.1 \pm 1.69^{\mathrm{y}}$ & $35.7 \pm 2.91^{\mathrm{y}}$ & $36.6 \pm 1.73^{y}$ & $34.7 \pm 2.79^{y}$ \\
\hline$S A F A$ & $23.5 \pm 5.59$ & $22.6 \pm 2.13$ & $23.1 \pm 1.32$ & $20.8 \pm 1.34$ & $21.8 \pm 1.73$ & $22.2 \pm 1.21$ \\
\hline$M U F A$ & $13.6 \pm 1.04^{\mathrm{zx}}$ & $14.5 \pm 1.50^{\mathrm{x}}$ & $12.6 \pm 0.82^{y}$ & $13.1 \pm 1.13^{\mathrm{y}}$ & $12.0 \pm 1.01^{\mathrm{z}}$ & $12.8 \pm 0.92^{\mathrm{y}}$ \\
\hline PUFA & $56.0 \pm 3.00^{\mathrm{z}}$ & $59.0 \pm 3.12^{\mathrm{y}}$ & $60.5 \pm 1.08^{\mathrm{yx}}$ & $61.1 \pm 2.36^{\mathrm{yx}}$ & $62.2 \pm 1.31^{\mathrm{x}}$ & $59.9 \pm 2.49^{\mathrm{yx}}$ \\
\hline$n 3$ & $52.8 \pm 2.85^{z}$ & $47.7 \pm 3.97^{\mathrm{y}}$ & $49.5 \pm 1.38^{\mathrm{zx}}$ & $49.7 \pm 3.09^{\mathrm{zyx}}$ & $51.3 \pm 1.33^{\mathrm{yx}}$ & $48.8 \pm 2.82^{\mathrm{yx}}$ \\
\hline$n 6$ & $3.17 \pm 0.33^{\mathrm{z}}$ & $11.6 \pm 1.04^{y}$ & $11.0 \pm 0.51^{\mathrm{y}}$ & $11.4 \pm 0.85^{\mathrm{y}}$ & $10.9 \pm 0.45^{\mathrm{y}}$ & $11.1 \pm 0.83^{\mathrm{y}}$ \\
\hline$n 3 / n 6$ & $16.8 \pm 1.53^{z}$ & $4.15 \pm 0.75^{\mathrm{y}}$ & $4.51 \pm 0.31^{\mathrm{y}}$ & $4.39 \pm 0.61^{\mathrm{y}}$ & $4.70 \pm 0.25^{\mathrm{y}}$ & $4.44 \pm 0.50^{\mathrm{y}}$ \\
\hline$n 3 H U F A / 18: 3 n 3$ & $77.8 \pm 9.57$ & $7.61 \pm 1.07$ & $6.73 \pm 0.64$ & $7.58 \pm 0.88$ & $7.89 \pm 0.37$ & $7.20 \pm 0.75$ \\
\hline
\end{tabular}




\begin{tabular}{|c|c|c|c|c|c|c|}
\hline & FO & SesO & MO & М 0.29 & M 0.58 & MO 1.16 \\
\hline \multicolumn{7}{|l|}{ Triacylglycerols } \\
\hline $14: 0$ & $4.62 \pm 0.40^{z}$ & $1.14 \pm 0.29^{\mathrm{y}}$ & $1.25 \pm 0.26^{\mathrm{y}}$ & $1.19 \pm 0.35^{\mathrm{y}}$ & $1.39 \pm 0.27^{\mathrm{y}}$ & $1.33 \pm 0.26^{\mathrm{y}}$ \\
\hline $16: 0$ & $12.0 \pm 0.18^{z}$ & $8.40 \pm 0.63^{\mathrm{y}}$ & $7.49 \pm 0.46^{\mathrm{x}}$ & $7.42 \pm 0.70^{\mathrm{x}}$ & $7.84 \pm 0.99^{\mathrm{yx}}$ & $7.55 \pm 0.43^{\mathrm{x}}$ \\
\hline $16: 1$ & $7.01 \pm 0.46^{\mathrm{z}}$ & $1.37 \pm 0.36^{\mathrm{y}}$ & $1.42 \pm 0.30^{\mathrm{y}}$ & $1.34 \pm 0.44^{\mathrm{y}}$ & $1.61 \pm 0.34^{\mathrm{y}}$ & $1.48 \pm 0.34^{\mathrm{y}}$ \\
\hline $18: 0$ & $2.85 \pm 0.23^{z}$ & $4.36 \pm 0.25^{\mathrm{y}}$ & $3.72 \pm 0.13^{\mathrm{x}}$ & $3.71 \pm 0.14^{\mathrm{x}}$ & $4.09 \pm 0.26^{\mathrm{v}}$ & $3.77 \pm 0.08^{\mathrm{x}}$ \\
\hline $18: \ln -9$ & $15.8 \pm 0.42^{z}$ & $25.1 \pm 2.58^{\mathrm{y}}$ & $20.8 \pm 0.51^{x}$ & $21.3 \pm 0.48^{\mathrm{x}}$ & $21.2 \pm 0.32^{\mathrm{x}}$ & $21.0 \pm 0.60^{\mathrm{x}}$ \\
\hline $18: 1 n-7$ & $3.02 \pm 0.21^{\mathrm{z}}$ & $2.06 \pm 1.76^{\mathrm{y}}$ & $1.26 \pm 0.08^{\mathrm{y}}$ & $1.22 \pm 0.14^{\mathrm{y}}$ & $1.37 \pm 0.16^{\mathrm{y}}$ & $1.28 \pm 0.11^{\mathrm{y}}$ \\
\hline $18: 1 n-5$ & $0.22 \pm 0.02^{\mathrm{zv}}$ & $0.08 \pm 0.07^{\mathrm{yx}}$ & $0.13 \pm 0.05^{\mathrm{zxv}}$ & $0.18 \pm 0.01^{\mathrm{vu}}$ & $0.11 \pm 0.09^{\mathrm{xu}}$ & $0.23 \pm 0.14^{\mathrm{v}}$ \\
\hline $18: 2 n-6$ & $4.13 \pm 0.64^{z}$ & $25.0 \pm 1.81^{\mathrm{y}}$ & $27.7 \pm 0.9^{x}$ & $27.2 \pm 1.65^{\mathrm{xy}}$ & $26.3 \pm 1.35^{\mathrm{xy}}$ & $26.5 \pm 1.52^{x y}$ \\
\hline $18: 3 n-3$ & $1.78 \pm 0.25^{\mathrm{z}}$ & $11.9 \pm 0.71^{\mathrm{y}}$ & $14.2 \pm 0.66^{\mathrm{x}}$ & $13.7 \pm 1.01^{\mathrm{v}}$ & $12.9 \pm 0.86^{\mathrm{xv}}$ & $13.6 \pm 1.29^{\mathrm{xv}}$ \\
\hline $18: 4 n-3$ & $2.68 \pm 0.53^{z}$ & $5.96 \pm 0.66^{\mathrm{y}}$ & $6.24 \pm 0.48^{y}$ & $6.57 \pm 1.20^{\mathrm{y}}$ & $5.89 \pm 0.78^{\mathrm{y}}$ & $6.47 \pm 0.19^{y}$ \\
\hline $20: 1$ & $3.78 \pm 1.31^{\mathrm{z}}$ & $1.09 \pm 0.28^{\mathrm{y}}$ & $1.13 \pm 0.06^{\mathrm{y}}$ & $1.10 \pm 0.29^{y}$ & $1.38 \pm 0.23^{\mathrm{y}}$ & $1.20 \pm 0.28^{\mathrm{y}}$ \\
\hline $20: 2 n-6$ & $0.32 \pm 0.04^{z}$ & $0.44 \pm 0.05^{\mathrm{y}}$ & $0.39 \pm 0.01^{\mathrm{y}}$ & $0.41 \pm 0.06^{\mathrm{y}}$ & $0.46 \pm 0.06^{\mathrm{y}}$ & $0.42 \pm 0.06^{\mathrm{y}}$ \\
\hline $20: 3 n-6$ & $0.14 \pm 0.05^{z}$ & $0.47 \pm 0.11^{\mathrm{y}}$ & $0.47 \pm 0.03^{\mathrm{y}}$ & $0.50 \pm 0.04^{\mathrm{y}}$ & $0.49 \pm 0.09^{\mathrm{y}}$ & $0.47 \pm 0.05^{\mathrm{y}}$ \\
\hline $20: 4 n-6$ & $0.62 \pm 0.07^{z}$ & $0.25 \pm 0.02^{\mathrm{y}}$ & $0.22 \pm 0.03^{\mathrm{y}}$ & $0.23 \pm 0.03^{\mathrm{y}}$ & $0.27 \pm 0.01^{\mathrm{y}}$ & $0.25 \pm 0.02^{\mathrm{y}}$ \\
\hline $20: 3 n-3$ & $0.14 \pm 0.09^{\mathrm{z}}$ & $0.26 \pm 0.04^{y}$ & $0.26 \pm 0.01^{\mathrm{y}}$ & $0.28 \pm 0.05^{\mathrm{y}}$ & $0.29 \pm 0.05^{\mathrm{y}}$ & $0.29 \pm 0.08^{\mathrm{y}}$ \\
\hline $20: 4 n-3$ & $6.03 \pm 0.49^{z}$ & $1.31 \pm 0.27^{\mathrm{y}}$ & $1.15 \pm 0.37^{\mathrm{y}}$ & $1.24 \pm 0.54^{\mathrm{y}}$ & $1.67 \pm 0.24^{\mathrm{y}}$ & $1.40 \pm 0.43^{\mathrm{y}}$ \\
\hline $22: 1$ & $0.41 \pm 0.06$ & $0.12 \pm 0.03$ & $0.27 \pm 0.36$ & $0.13 \pm 0.03$ & $0.16 \pm 0.02$ & $0.14 \pm 0.03$ \\
\hline $20: 5 n-3$ & $6.05 \pm 0.43^{z}$ & $1.31 \pm 0.18^{\mathrm{y}}$ & $1.41 \pm 0.21^{\mathrm{y}}$ & $1.29 \pm 0.30^{\mathrm{y}}$ & $1.48 \pm 0.13^{\mathrm{y}}$ & $1.47 \pm 0.28^{\mathrm{y}}$ \\
\hline $24: 1$ & $0.56 \pm 0.05^{z}$ & $0.19 \pm 0.03^{\mathrm{y}}$ & $0.21 \pm 0.04^{\mathrm{y}}$ & $0.19 \pm 0.04^{\mathrm{y}}$ & $0.24 \pm 0.04^{\mathrm{y}}$ & $0.21 \pm 0.02^{y}$ \\
\hline $22: 5 n-3$ & $2.90 \pm 0.24^{z}$ & $0.71 \pm 0.17^{\mathrm{y}}$ & $0.79 \pm 0.17^{\mathrm{yx}}$ & $0.71 \pm 0.17^{\mathrm{y}}$ & $0.95 \pm 0.10^{\mathrm{x}}$ & $0.83 \pm 0.17^{\mathrm{yx}}$ \\
\hline $22: 6 n-3$ & $17.1 \pm 0.92^{z}$ & $4.20 \pm 0.59^{\mathrm{y}}$ & $4.09 \pm 0.49^{\mathrm{y}}$ & $4.00 \pm 0.84^{\mathrm{y}}$ & $4.73 \pm 0.41^{\mathrm{y}}$ & $4.51 \pm 0.85^{\mathrm{y}}$ \\
\hline$S A F A$ & $19.7 \pm 0.42^{z}$ & $14.3 \pm 0.97^{\mathrm{x}}$ & $12.9 \pm 1.35^{\mathrm{y}}$ & $12.7 \pm 1.13^{\mathrm{y}}$ & $13.7 \pm 1.40^{\mathrm{yx}}$ & $13.05 \pm 0.72^{y}$ \\
\hline MUFA & $30.9 \pm 0.65^{z}$ & $30.0 \pm 3.32^{\mathrm{x}}$ & $25.6 \pm 0.60^{y}$ & $25.6 \pm 0.51^{y}$ & $26.1 \pm 0.95^{\mathrm{y}}$ & $25.67 \pm 0.85^{y}$ \\
\hline PUFA & $41.9 \pm 1.55^{z}$ & $52.3 \pm 2.52^{x}$ & $56.8 \pm 1.40^{\mathrm{y}}$ & $56.4 \pm 1.89^{\mathrm{y}}$ & $55.8 \pm 1.53^{y}$ & $56.2 \pm 1.73^{\mathrm{y}}$ \\
\hline$n 3$ & $36.7 \pm 1.40^{z}$ & $25.6 \pm 1.04^{\mathrm{x}}$ & $28.1 \pm 0.65^{\mathrm{y}}$ & $27.8 \pm 1.08^{\mathrm{y}}$ & $27.9 \pm 0.76^{\mathrm{y}}$ & $28.6 \pm 1.04^{y}$ \\
\hline$n 6$ & $5.25 \pm 0.68^{z}$ & $26.7 \pm 1.71^{\mathrm{y}}$ & $28.7 \pm 0.85^{\mathrm{x}}$ & $28.5 \pm 1.60^{y}$ & $28.0 \pm 0.86^{\mathrm{yx}}$ & $27.6 \pm 1.57^{\mathrm{yx}}$ \\
\hline$n 3 / n 6$ & $7.07 \pm 0.88^{z}$ & $0.96 \pm 0.05^{\mathrm{y}}$ & $0.98 \pm 0.02^{\mathrm{y}}$ & $0.98 \pm 0.07^{\mathrm{y}}$ & $1.00 \pm 0.02^{\mathrm{y}}$ & $1.04 \pm 0.08^{\mathrm{y}}$ \\
\hline$n-3 H U F A / 18: 3 n 3$ & $19.9 \pm 3.24^{z}$ & $1.17 \pm 0.14^{\mathrm{y}}$ & $0.99 \pm 0.07^{y}$ & $1.04 \pm 0.18^{\mathrm{y}}$ & $1.17 \pm 0.09^{\mathrm{y}}$ & $1.11 \pm 0.19^{y}$ \\
\hline
\end{tabular}

Abbreviations: see Table $2, \mathrm{n}-3$ HUFA $=\mathrm{n}-3$ highly unsaturated fatty acids. ${ }^{\mathrm{u}-\mathrm{z}}$ Mean values across the row not sharing a common superscript are significantly different by $P<0.05$.

Table 6. Content of vitamin $\mathrm{E}$, sesamin and episesamin in white muscle and liver $\left(\mu \mathrm{g} \mathrm{g}^{-1}\right)$.

\begin{tabular}{|c|c|c|c|c|c|c|}
\hline & FO & SesO & MO & MO 0.29 & MO 0.58 & MO 1.16 \\
\hline \multicolumn{7}{|l|}{ White muscle } \\
\hline$\alpha$-Tocopherol & $1.36 \pm 0.58$ & $1.00 \pm 0.19$ & $1.09 \pm 0.73$ & $1.46 \pm 0.20$ & $1.50 \pm 0.26$ & $1.53 \pm 0.23$ \\
\hline$\gamma$-Tocopherol & $0.15 \pm 0.04^{\mathrm{u}}$ & $0.80 \pm 0.50^{\mathrm{uv}}$ & $1.01 \pm 0.97^{\mathrm{vz}}$ & $1.74 \pm 0.36^{\mathrm{xy}}$ & $1.14 \pm 0.61^{\mathrm{vyz}}$ & $1.87 \pm 0.36^{\mathrm{x}}$ \\
\hline Episesamin & - & $0.32 \pm 0.23$ & - & $0.40 \pm 0.21$ & $0.37 \pm 0.21$ & $0.32 \pm 0.10$ \\
\hline Sesamin & - & $0.20 \pm 0.09$ & - & $0.20 \pm 0.12$ & $0.14 \pm 0.08$ & $0.16 \pm 0.04$ \\
\hline \multicolumn{7}{|l|}{ Liver } \\
\hline$\alpha$-Tocopherol & $87.8 \pm 37.7^{\mathrm{y}}$ & $146.4 \pm 28.3^{y}$ & $234.7 \pm 83.5^{z}$ & $195.5 \pm 56.4^{z}$ & $231.74 \pm 87.8^{z}$ & $188.0 \pm 63.6^{\mathrm{Z}}$ \\
\hline$\gamma$-Tocopherol & $1.73 \pm 1.29^{\mathrm{y}}$ & $11.9 \pm 4.05^{\mathrm{z}}$ & $11.3 \pm 3.00^{\mathrm{z}}$ & $8.30 \pm 2.30^{\mathrm{z}}$ & $9.46 \pm 3.11^{\mathrm{z}}$ & $9.37 \pm 2.63^{z}$ \\
\hline Episesamin & - & $2.43 \pm 0.95^{\mathrm{yz}}$ & - & $1.69 \pm 0.78^{y}$ & $2.32 \pm 0.52^{\mathrm{yz}}$ & $3.14 \pm 0.75^{\mathrm{z}}$ \\
\hline Sesamin & - & $1.90 \pm 1.17^{\mathrm{x}}$ & - & $0.67 \pm 0.27^{z}$ & $0.81 \pm 0.08^{\mathrm{zy}}$ & $1.53 \pm 0.43^{\mathrm{xy}}$ \\
\hline
\end{tabular}

Abbreviations: see Table 2. MO, $n=4 ; \mathrm{FO}, \mathrm{n}=3$; SesO, $n=4 ;$ MO $0.29 \mathrm{n}=5 ;$ MO $0.58 \mathrm{n}=4$; MO $1.16 \mathrm{n}=5$. ${ }^{\text {u-z }}$ Mean values across the row not sharing a common superscript are significantly different by $P<0.05$. 


\section{Tocopherols and sesamin content}

The contents of $\alpha$ - and $\gamma$ - tocopherols in the liver were significantly lower $(P<0.05)$ in FO group compared to the MO groups with or without addition of sesamin. In the white muscle, the $\gamma$-tocopherol content was significantly lower in the FO group than in the MO groups $(P<0.05)$. No differences were found in the content of sesamin and episesamin in white muscle, in the liver sesamin and episesamin levels were higher in the MO 1.16 group, the difference was significant compared to the MO 0.29 group $(P=0.03, P<0.01$ for sesamin and episesamin respectively), but was higher than in the $\mathrm{Ses} O$ ( $P=0.3, P=0.2$ for sesamin and episesamin respectively) and $\mathrm{MO} 0.58$ ( $P=0.1$ both for sesamin and episesamin) group as well (Table 6).

\section{Relative expression of target genes}

The relative expressions of target genes in the experimental groups, compared to MO group, after normalization to the reference gene are shown in Figure 1. In the MO 1.16 group, $\operatorname{PPAR} \alpha(P=0.05)$ was upregulated compared to control group. In the MO 0.29 group, SRB $(P=0.03)$ and HSL3 $(P<0.01)$ were upregulated, and in the MO 0.58 group, HSL3 $(P=0.03)$ was upregulated compared to the MO group.

\section{Total content of CYP and EROD activity in the liver}

The EROD activity did not differ significantly among groups (Figure 2). The CYP (Figure 2) levels were significantly higher in the MO 0.29 group $(P=0.02)$ and slightly higher in the MO 1.16 group $(P=0.07)$ compared to $\mathrm{MO}$ group and significantly lower in $\mathrm{MO}$ 0.58 group $(P<0.01)$ compared to MO 0.29 group. The CYP response was also significantly higher in the $\mathrm{SesO}$ group compared to the MO group. There was a large variation in individual response detected, with $\mathrm{CV}$ values within groups from 16 up to $125 \%$.

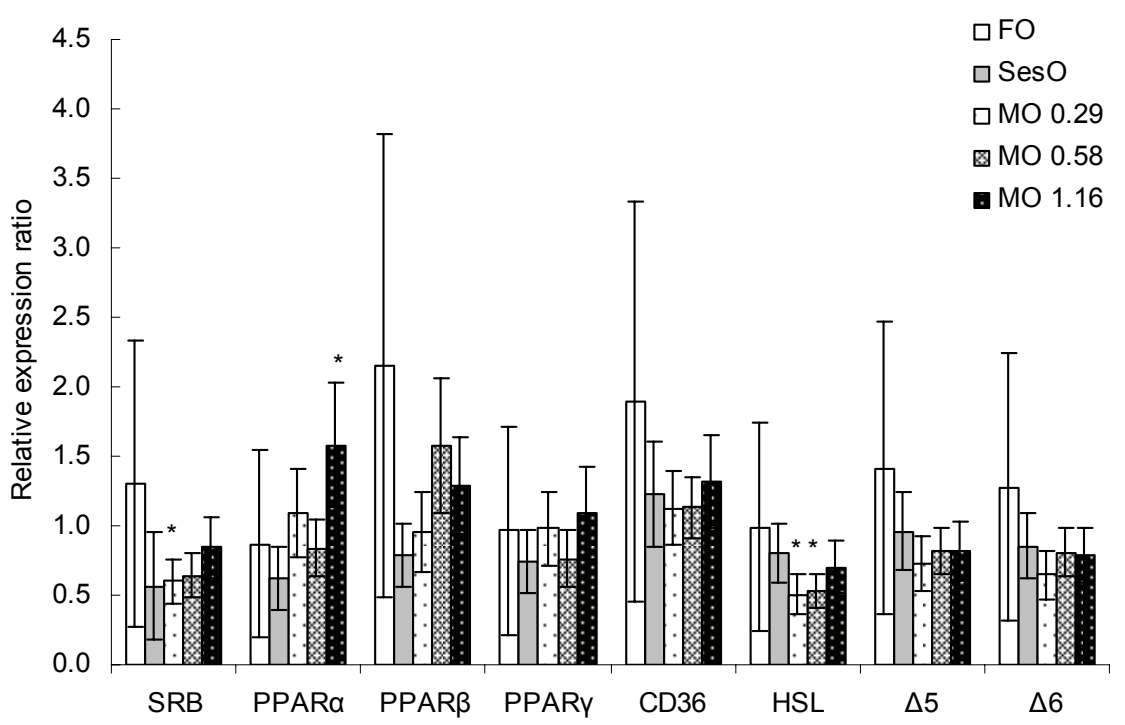

Fig. 1. Relative expression ratio compared to the MO group of the analyzed genes in the $\mathrm{FO}$, SesO, MO 0.29 , MO 0.58 and MO 1.16 groups. Genes with significantly different expression ratio to the MO group are indicated with an asterisk $(P<0.05)$. For abbreviations see Table 2 and 3.
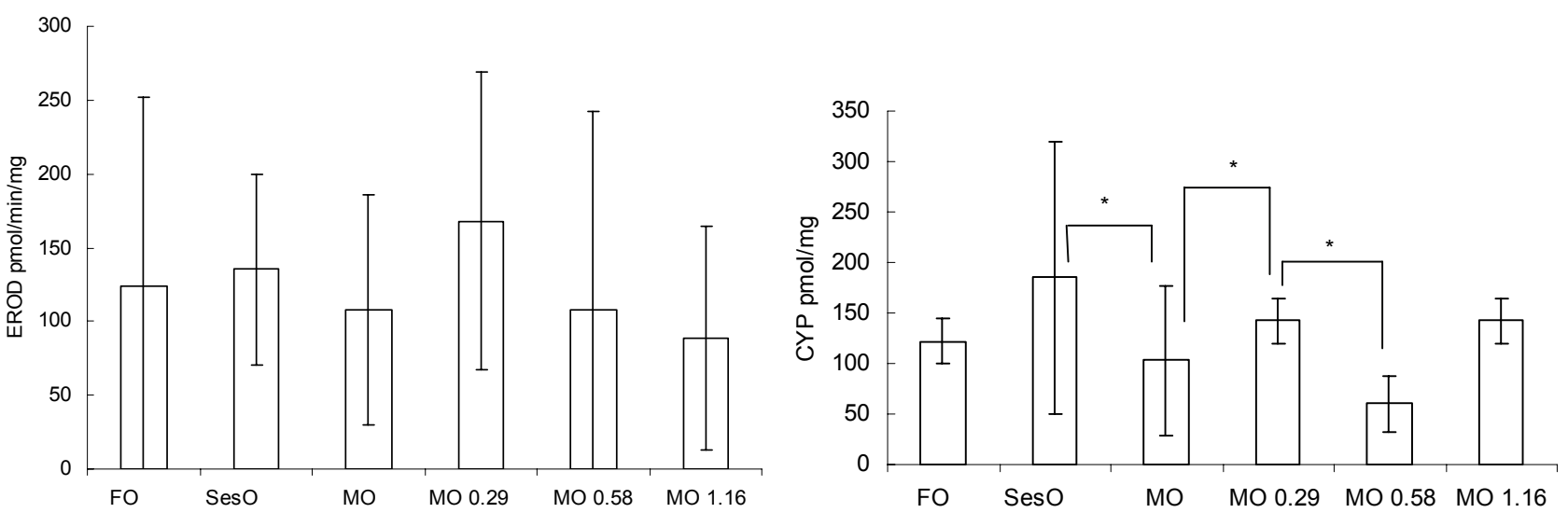

Fig. 2. Ethoxyresorufin O-deethylase activity, EROD (pmol. $\mathrm{min}^{-1} \cdot \mathrm{mg}^{-1}$ protein) and total cytochrome P450, CYP (nM/mg microsomal protein) in the liver. MO, $n=4 ; F O, n=3$; SesO, $n=4 ; M O 0.29 n=5 ; M O 0.58 n=4 ; M O 1.16 n=5$. Significantly different CYP content are indicated with an asterisk $(P<0.05)$. 


\section{Discussion}

In general, the results of different analyses in this study showed a large variation caused by a broad individual response. The reason for the different weight in the fish group is most likely caused by the low number of individuals as the fish were divided between groups according to stastistical methods. In Sweden, the long breeding history of Baltic Atlantic salmon smolts is aimed for release purposes as a replacement for the wild spawners being hindered the enter the rivers by hydroelectric power dams and the large number of parents is a prerequisite. Therefore, this fish had a wide range of genetic background compared to the fish used in our previous studies on rainbow trout and Atlantic salmon hepatocytes. Schlechtriem et al. (2007) found inter individual variations in FA composition of Atlantic salmon smolt, and suggested individual variation in elongation and desaturation capacity as a likely explanation. Other known factors affecting the capacity of elongation and desaturation are environmental factors and life stage, e.g. salmon prior seawater transfer had higher relative expression of $\Delta-5$ and $\Delta-6$ desaturase (Zheng et al. 2005). Addition of bioactive compounds such as 3-thia fatty acids, conjugate linolenic acid and lipoic acid can also influence lipid metabolism. The 3 -thia fatty acids increased $\beta$-oxidation capacity and the levels of DHA (Moya-Falcon et al. 2006) and reduced mRNA expression of PPAR $\alpha$ and apolipoproteinAI (ApoAI) (Kleveland et al. 2006) in Atlantic salmon. Conjugated linoleic acid decreased adipocytes by elevating energy expenditure (Kennedy et al. 2009). Dietary lipoic acid was shown to increase EPA levels in pacu (Piractus mesopotamicus) muscle (Trattner et al. 2007).

In our previous study on rainbow trout, significantly increased levels of DHA and decreased proportions of 18:3n-3 in the TAG and PL fractions were found after sesamin addition to the fish diet. Also the total level of polyunsaturated fatty acids (PUFA) was decreased, possibly due to increased $\beta$-oxidation of PUFA in sesamin fed fish (Trattner et al. 2008a). These results were confirmed in an in vitro study on Atlantic salmon hepatocytes incubated with radiolabelled 18:3n-3 with or without sesamin addition to the media. It was shown that the amount of 18:3n-3 elongated and desaturated to DHA was increased after sesamin incubation. It was also shown that sesamin increased the total level of $\beta$-oxidation products, in particular acetate, which indicated peroxisomal $\beta$-oxidation (Trattner et al. 2008b). Sesamin also decreased secretion of lipids (mainly TAG) in Atlantic salmon hepatocytes, in agreement with the lipid lowering effects reported as reduced TAG and VLDL levels in rat serum (UmedaSawada et al. 1998, Kamal-Eldin et al. 2000). In the present study, sesamin significantly decreased levels of 18:3n-3 in the PL of all sesamin fed groups, also the average DHA level was (not significantly) increased in the groups after sesamin addition to the diet.

In the rainbow trout study, two different oils, sunflower: linseed oil mixture (MO) and linseed oil (LO) were used. In that study the effects of sesamin on gene expression and FA composition were greater in MO diet than in LO diet. The use of $100 \%$ linseed oil in the LO group decreased desaturation index (n-3HUFA/18:3n-3) compared to the use of a mixture of vegetable oil as in the MO group, indicating less efficient conversion of 18:3n-3 to DHA when linseed oil is included at higher levels in the diet. There are a number of studies showing decreased desaturation index with increased inclusion of linseed oil, a summary of results from studies are presented in Table 7. In a study on Atlantic salmon, it was suggested that increased inclusion of linseed oil due to its high content of 18:3n-3 inhibited elongation and desaturation of $18: 3 n-3$ in hepatocytes, and increased oxidation of $18: 3 n-$ 3 in enterocytes (Tocher et al. 2002). The replacement of fish oil with linseed oil, decreased the DHA levels in the liver 4-fold (Tocher et al. 2002), whereas replacement of the fish oil with rapeseed oil decreased the DHA levels to half (Bell et al. 2001). (Leaver et al. 2008) showed increased activity of fatty acyl $\Delta-6$ desaturase in the liver of Atlantic salmon fed vegetable oils, the increased activity was highest for rapeseed oil followed by soybean oil and last linseed oil. The expression of elov15b and elovl2 elongases were also significantly higher in liver of vegetable oil fed fish compared to fish oil fed fish, with lower expression in linseed oil fed fish than in rapeseed oil and soybean oil fed fish (Morais et al. 2009). In agreement with these studies, we also found decreased desaturation index in the MO group, which had higher 18:3n-3 content in the diet compared to the $\mathrm{Ses} O$ group.

The differences in tocopherols (Table 6) are due to the difference in tocopherols in the diet, the vegetable oil had a higher content of tocopherols than the fish oil. The FO diet had low levels of $\alpha$-tocopherol and levels below detection limit for $\gamma$-tocopherol. In contrast to our results, in rats and humans, it has been shown that sesamin increased the levels of $\gamma$-tocopherol and reduced 
Table 7. An overview of the desaturation index in Atlantic salmon and rainbow trout fed different oils.

\begin{tabular}{|c|c|c|c|c|c|c|}
\hline Reference & $\begin{array}{l}\text { 18:3n3 } \\
\text { (\%) diet }\end{array}$ & $\begin{array}{l}\text { Oil } \\
\text { source }\end{array}$ & $\begin{array}{l}\text { Desat. } \\
\text { index }\end{array}$ & Species & Tissue & Lipid fraction \\
\hline \multirow{4}{*}{$\begin{array}{l}\text { Leaver et al. } \\
(2008)\end{array}$} & 1.2 & $\mathrm{FO}$ & 77.2 & A. salmon & Liver & Total lipid \\
\hline & 44.9 & LO & 1.3 & & & \\
\hline & 8.1 & RO & 11.5 & & & \\
\hline & 5.7 & $\mathrm{SO}$ & 8.6 & & & \\
\hline \multirow{8}{*}{$\begin{array}{l}\text { Menoyo et al. } \\
\text { (2007) }\end{array}$} & 41.7 & LO 100 & 1.73 & A. salmon & Muscle & Polar lipids \\
\hline & 30.2 & LO 75 & 2.71 & & & \\
\hline & 25.8 & LO 50 & 2.85 & & & \\
\hline & 12.1 & LO 25 & 5.20 & & & \\
\hline & 41.7 & LO 100 & 0.44 & A. salmon & Muscle & Neutral lipids \\
\hline & 30.2 & LO 75 & 0.56 & & & \\
\hline & 25.8 & LO 50 & 0.65 & & & \\
\hline & 12.1 & LO 25 & 1.27 & & & \\
\hline \multirow{4}{*}{$\begin{array}{l}\text { Trattner et al. } \\
(2008 \mathrm{a})\end{array}$} & 31.7 & MO & 10.4 & R. trout & Muscle & Phospholipid \\
\hline & 53.4 & LO & 5.0 & & & \\
\hline & 31.7 & MO & 1.3 & & & Triacylglycerol \\
\hline & 53.4 & LO & 0.7 & & & \\
\hline \multirow{3}{*}{$\begin{array}{l}\text { Rosenlund et al. } \\
\text { (2001) }\end{array}$} & 10.2 & RO & 1.8 & A. salmon & Muscle & Total lipid \\
\hline & 22.6 & LO & 0.9 & & & \\
\hline & 17.0 & $\mathrm{SO}$ & 1.0 & & & \\
\hline
\end{tabular}

Desat. index $=$ Desaturation index $=(n-3 H U F A>18 C) / 18: 3 n-3 ; F O=$ fish oil, $L O=$ linseed oil, RO = rapeseed oil, SO = soybean oil, LO100 $=100 \%$ linseed oil, LO75 = $75 \%$ linseed oil and $25 \%$ sunflower oil, LO50 = 50\% linseed oil and $50 \%$ sunflower oil, LO25= $25 \%$ linseed oil and $75 \%$ sunflower oil, MO = mixture of linseed oil and sunflower oil (6:4).

the urine extraction of its metabolites (Frank et al. 2004, Kamal-Eldin et al. 1995). In the liver, the sesamin and episesamin contents were increased with increased content in the diet. Even though the sesamin : episesamin ratio was 1:1 in the feed, episesamin was detected at higher levels in muscle and liver. This finding is in agreement with the study on rainbow trout and has also been found in rats (Trattner et al. 2008a, Umeda-Sawada et al. 1999).

Interestingly, even if there were no significant differences in the composition of n-3 HUFA in TAG, it was found that the MO $1.16 \mathrm{~g}$ group with increased relative expression of PPAR $\alpha$ also had higher proportions of DPA and DHA in white muscle PL and TAG. A result well corresponding with the increased levels of DHA in the in vivo rainbow trout livers and the in vitro Atlantic salmon hepatocytes and increase $\beta$-oxidation in Atlantic salmon hepatocytes. The relative expression of PPAR $\alpha$ in rainbow trout liver and Atlantic salmon hepatocytes, was also found previously to be effected by sesamin, although in these previous cases, this expression was downregulated. The highest levels of DPA and DHA were found in PL and TAG of the MO 0.58 group, which also had increased relative expression of HSL3. HSL activate intracellular hydrolysis of TAG, which can then be used for $\beta$-oxidation (Watt et al. 2003). The increase in DHA could also indicate increased $\beta$-oxidation as DHA is produced through $\beta$-oxidation of longer n-3 fatty acids (Voss et al. 1991).

The average values for each group in the EROD analysis indicate that the activity decreases with increased levels of sesamin and for the CYP analysis the content is lower at intermediate doses than at low and high dose of sesamin (Figure 2). However, due to the large variation within groups, it is difficult to draw any conclusions. It would be interesting to study the dose dependent response under in vitro conditions. The large individual response of enzymes involved in the defence against 
xenobiotic compounds also support the above mentioned suggestion that individual fish react differently to bioactive compounds in the diet, also in terms of lipid metabolism.

To improve lipid metabolism in farmed fish may be a useful tool in the future to meet the demands for production of farmed fish with a healthy FA composition produced on less amounts of fish based raw materials. This study indicates that the response of Atlantic salmon (Swedish Baltic origin) to sesamin is less than in rainbow trout in vivo (Trattner et al. 2008a) and in Atlantic salmon in vitro (Trattner et al. 2008b). This difference deserves further evaluation and may be utilised for selection to improve desaturation capacity in farmed fish fed vegetable oils.

\section{Conflict of Interest}

There is no conflict of interest.

\section{Abbreviations}

$\begin{array}{ll}\text { ACO } & \text { Acyl-CoA oxidase } \\ \text { CD 36 } & \text { Cluster of differentiation } 36 \\ \text { CPT } & \text { Carnitine palmitoyltransferase } \\ \text { CYP } & \text { Cytochrome P450 }\end{array}$

$\begin{array}{ll}\Delta 5 & \Delta 5 \text { desaturase } \\ \Delta 6 & \Delta 6 \text { desaturase } \\ \text { DHA } & \text { Docosahexaenoic acid (22:6n-3) } \\ \text { DPA } & \text { Docosapentaenoic acid }(22: 5 n-3) \\ \text { EF1A } & \text { Elongation factor } 1 \alpha \\ \text { EPA } & \text { Eicosapentaenoic acid (20:5n-3) } \\ \text { EROD } & \text { Ethoxyresorufin O-deethylase } \\ \text { FA } & \text { Fatty acid } \\ \text { FO } & \text { Fish oil } \\ \text { HSL3 } & \text { Hormone sensitive lipase } \\ \text { HUFA } & \text { Highly unsaturated fatty acids } \\ \text { MO } & \text { Linseed and sunflower oil } 6: 4 \text { by vol } \\ \text { MUFA } & \text { Monounsaturated fatty acids } \\ \text { PL } & \text { Phospholipids } \\ \text { TAG } & \text { Triacylglycerol } \\ \text { TLC } & \text { Thin-layer chromatography } \\ \text { SesO } & \text { Sesame oil and linseed oil 1:1 by vol } \\ \text { SRB-I } & \text { Scavenger receptor type B } \\ \text { RPL2 } & \text { RNA polymerase II polypeptide } \\ \text { PCR } & \text { Polymerase chain reaction } \\ \text { PPAR } & \text { Peroxisome proliferator-activated receptor } \\ \text { PUFA } & \text { Polyunsaturated fatty acids } \\ \text { SREBP } & \text { Sterol regulatory element binding protein } \\ \text { VLDL } & \text { Very low-density lipoprotein }\end{array}$

\section{References}

APPELQVIST L: Rapid methods of lipid extractions and fatty acid methyl ester preparation for seed and leaf tissue with special remarks on preventing the accumulation of lipids contaminants. Royal Swedish Academy of Science (Kungliga Svenska Vetenskapsakademien) 28: 551-570, 1968.

ASHAKUMARY L, ROUYER I, TAKAHASHI Y, IDE T, FUKUDA N, AOYAMA T, HASHIMOTO T, MIZUGAKI M, SUGANO M: Sesamin, a sesame lignan, is a potent inducer of hepatic fatty acid oxidation in the rat. Metabolism-Clin Exp 48: 1303-1313, 1999.

BELL JG, MCEVOY J, TOCHER DR, MCGHEE F, CAMPBELL PJ, SARGENT JR: Replacement of fish oil with rapeseed oil in diets of Atlantic salmon (Salmo salar) affects tissue lipid compositions and hepatocyte fatty acid metabolism. $J$ Nutr 131: 1535-1543, 2001.

BERGE K, TRONSTAD KJ, FLINDT EN, RASMUSSEN TH, MADSEN L, KRISTIANSEN K, BERGE RK: Tetradecylthioacetic acid inhibits growth of rat glioma cells ex vivo and in vivo via PPAR-dependent and PPAR-independent pathways. Carcinogenesis 22: 1747-1755, 2001.

FAO 2007 FAOSTAT, faostat.fao.org/. (8 November 2007).

FRANK J, KAMAL-ELDIN A, TRABER MG: Consumption of sesame oil muffins decreases the urinary excretion of gamma-tocopherol metabolites in humans. Vitamin E Health 1031: 365-367, 2004.

HARA A, RADIN NS: Lipid extraction of tissue with low toxicity solvent. Anal Biochem 90: 420-426, 1978.

HAVELKOVA M, RANDAK T, ZLABEK V, KRIJT J, KROUPOVA H, PULKRABOVA J, SVOBODOVA Z: Biochemical markers for assessing aquatic contamination. Sensors 7: 2599-2611, 2007.

HUONG DTT, IDE T: Dietary lipoic acid-dependent changes in the activity and mRNA levels of hepatic lipogenic enzymes in rats. Br J Nutr 100: 79-87, 2008.

IDE T, HONG DD, RANASINGHE P, TAKAHASHI Y, KUSHIRO M, SUGANO M: Interaction of dietary fat types and sesamin on hepatic fatty acid oxidation in rats. Biochim Biophys Acta 1682: 80-91, 2004. 
JENG KCG, HOU RCW: Sesamin and sesamolin: nature's therapeutic lignans. Curr Enzyme Inhib 1: 11-20, 2005.

JÖNSSON M, ABRAHAMSON A, BRUNSTRÖM B, BRANDT I: Cytochrome P4501A induction in rainbow trout gills and liver following exposure to waterborne indigo, benzo(a)pyrene and 3,3',4',5-pentachlorobiphenyl. Aquatic Toxicol 79: 226-232, 2006.

KAMAL-ELDIN A, FRANK J, RAZDAN A, TENGBLAD S, BASU S, VESSBY B: Effects of dietary phenolic compounds on tocopherol, cholesterol, and fatty acids in rats. Lipids 35: 427-435, 2000.

KAMAL-ELDIN A, PETTERSSON D, APPELQVIST LA: Sesamin (a compound from sesame oil) increases tocopherol levels in rats fed ad-libitum. Lipids 30: 499-505, 1995.

KISO Y, TSURUOKA N, KIDOKORO A, MATSUMOTO I, ABE K: Sesamin ingestion regulates the transcription levels of hepatic metabolizing enzymes for alcohol and lipids in rats. Alcoholism Clin Exp Res 29: 116S-120S, 2005.

KLEVELAND EJ, RUYTER B, VEGUSDAL A, SUNDVOLD H, BERGE RK, GJOEN T: Effects of 3-thia fatty acids on expression of some lipid related genes in Atlantic salmon (Salmo salar L.). Comp Biochem Physiol B 145: 239-248, 2006.

KUSHIRO M, MASAOKA T, HAGESHITA S, TAKAHASHI Y, IDE T, SUGANO M: Comparative effect of sesamin and episesamin on the activity and gene expression of enzymes in fatty acid oxidation and synthesis in rat liver. J Nutr Biochem 13: 289-295, 2002.

LEAVER MJ, VILLENEUVE LAN, OBACH A, JENSEN L, BRON JE, TOCHER DR, TAGGART JB: Functional genomics reveals increases in cholesterol biosynthetic genes and highly unsaturated fatty acid biosynthesis after dietary substitution of fish oil with vegetable oils in Atlantic salmon (Salmo salar). BMC Genomics 9: 299, 2008.

MOAZZAMI A, KAMAL-ELDIN A: Sesame seed is a rich source of dietary lignans. $J$ Am Oil Chem Soc 83: 719-723, 2006.

MORAIS S, MONROIG O, ZHENG XZ, LEAVER MJ, TOCHER DR: Highly unsaturated fatty acid synthesis in Atlantic salmon: characterization of ELOVL5-and ELOVL2-like elongases. Marine Biotechnol 11: 627-639, 2009.

MOYA-FALCON C, HVATTUM E, TRAN TN, THOMASSEN MS, SKORVE J, RUYTER B: Phospholipid molecular species, beta-oxidation, desaturation and elongation of fatty acids in Atlantic salmon hepatocytes: effects of temperature and 3-thia fatty acids. Comp Biochem Physiol B 145: 68-80, 2006.

MOZAFFARIAN D, RIMM EB: Fish intake, contaminants, and human health - evaluating the risks and the benefits. JAMA 296: 1885-1899, 2006.

MURRAY M: Mechanisms of inhibitory and regulatory effects of methylenedioxyphenyl compounds on cytochrome P450-dependent drug oxidation. Curr Drug Metab 1: 67-84, 2000.

NELSON DR, KOYMANS L, KAMATAKI T, STEGEMAN JJ, FEYEREISEN R, WAXMAN DJ, WATERMAN MR, GOTOH O, COON MJ, ESTABROOK RW, GUNSALUS IC, NEBERT DW: P450 superfamily: update on new sequences, gene mapping, accession numbers and nomenclature. Pharmacogenetics 6: 1-42, 1996.

OMURA T, SATO R: The carbon monoxide-binding pigment of liver microsomes. II. Solubilization, purification, and properties. J Biol Chem 239: 2379-2385, 1964.

PETTERSSON A, JOHNSSON L, BRANNAS E, PICKOVA J: Effects of rapeseed oil replacement in fish feed on lipid composition and self-selection by rainbow trout (Oncorhynchus mykiss). Aquacult Nutr 15: 577-586, 2009.

PFAFFL MW, HORGAN GW, DEMPFLE L: Relative expression software tool (REST (c)) for group-wise comparison and statistical analysis of relative expression results in real-time PCR. Nucleic Acids Res 30, 2002.

SCHLECHTRIEM C, BRON JE, TOCHER DR: Inter-individual variation in total fatty acid compositions of flesh of Atlantic salmon smolts-fed diets containing fish oil or vegetable oil. Aquacult Res 38: 1045-1055, 2007.

SHIMIZU S, AKIMOTO K, SHINMEN Y, KAWASHIMA H, SUGANO M, YAMADA H: Sesamin is a potent and specific inhibitor of delta-5 desaturase in polyunsaturated fatty-acid biosynthesis. Lipids 26: 512-516, 1991.

SMITH PK, KROHN RI, HERMANSSON GT, MALILIA AK, GARTNER FH, PROVENZANO MD, FUJIMITO EK, GOEKE NM, OLSON BJ, KLENK DC: Measurement of protein using bicinchoninic acid. Anal Biochem 150: 76-85, 1985. 
TACON AGJ: Global trends in aquaculture and compound aquafeed production. In: International Aquafeed Directory and Buyers' Guide. AGJ TACON (ed), R A I, Turret Uxbridge, Middlesex (UK), 2005, pp 8-23.

TOCHER DR, FONSECA-MADRIGAL J, BELL JG, DICK JR, HENDERSON RJ, SARGENT JR: Effects of diets containing linseed oil on fatty acid desaturation and oxidation in hepatocytes and intestinal enterocytes in Atlantic salmon (Salmo salar). Fish Physiol Biochem 26: 157-170, 2002.

TORSTENSEN BE, BELL JG, ROSENLUND G, HENDERSON RJ, GRAFF IE, TOCHER DR, LIE O, SARGENT JR: Tailoring of Atlantic salmon (Salmo salar L.) flesh lipid composition and sensory quality by replacing fish oil with a vegetable oil blend. J Agric Food Chem 53: 10166-10178, 2005.

TRATTNER S, PICKOVA J, PARK KH, RINCHARD J, DABROWSKI K: Effects of alpha-lipoic and ascorbic acid on the muscle and brain fatty acids and antioxidant profile of the South American pacu Piaractus mesopotamicus. Aquaculture 273: 158-164, 2007.

TRATTNER S, KAMAL-ELDIN A, BRANNAS E, MOAZZAMI A, ZLABEK V, LARSSON P, RUYTER B, GJOEN T, PICKOVA J: Sesamin supplementation increases white muscle docosahexaenoic acid (DHA) levels in rainbow trout (Oncorhynchus mykiss) fed high alpha-linolenic acid (ALA) containing vegetable oil: metabolic actions. Lipids 43: 989-997, 2008a.

TRATTNER S, RUYTER B, OSTBYE TK, GJOEN T, ZLABEK V, KAMAL-ELDIN A, PICKOVA J: Sesamin increases alpha-linolenic acid conversion to docosahexaenoic acid in Atlantic salmon (Salmo salar L.) hepatocytes: role of altered gene expression. Lipids 43: 999-1008, 2008b.

UMEDA-SAWADA R, OGAWA M, IGARASHI O: The metabolism and n-6/n-3 ratio of essential fatty acids in rats: effect of dietary arachidonic acid and mixture of sesame lignans (sesamin and episesamin). Lipids 33: 567-527, 1998.

UMEDA-SAWADA R, OGAWA M, IGARASHI O: The metabolism and distribution of sesame lignans (sesamin and episesamin) in rats. Lipids 34: 633-637, 1999.

WATT MJ, STEINBERG GR, HEIGENHAUSER GJF, SPRIET LL, DYCK DJ: Hormone-sensitive lipase activity and triacylglycerol hydrolysis are decreased in rat soleus muscle by cyclopiazonic acid. Am J Physiol 285: E412E419, 2003.

WILLIAMS DE, LECH JJ, BUHLER DR: Xenobiotics and xenoestrogens in fish: modulation of cytochrome P450 and carcinogenesis. Mutat Res 399: 179-192, 1998.

VOSS A, REINHART M, SANKARAPPA S, SPRECHER H: The metabolism of 7,10,13,16,19-docosapentaenoic acid to 4,7,10,13,16,19-docosahexaenoic acid in rat-liver is independent of a 4-desaturase. J Biol Chem 266: 1999520000, 1991.

ZHENG XZ, TORSTENSEN BE, TOCHER DR, DICK JR, HENDERSON RJ, BELL JG: Environmental and dietary influences on highly unsaturated fatty acid biosynthesis and expression of fatty acyl desaturase and elongase genes in liver of Atlantic salmon (Salmo salar). Biochim Biophys Acta 1734: 13-24, 2005. 